\title{
Autogenous fat grafting for mild-to-moderate postoperative temporal hollowing after decompressive craniectomy: One-year follow-up
}

\author{
Jaemin Choi $^{1}$, Hyungon Choi ${ }^{1}$, Donghyeok Shin ${ }^{1}$, Jeenam Kim ${ }^{1}$, Myungchul Lee ${ }^{1}$, \\ Soonheum Kim ${ }^{2}$, Dongin $\mathrm{Jo}^{2}$, Cheolkeun Kim ${ }^{2}$ \\ ${ }^{1}$ Department of Plastic and Reconstructive Surgery, Konkuk University Medical Center, Seoul; ${ }^{2}$ Department of Plastic and Reconstructive \\ Surgery, Konkuk University Choongju Hospital, Konkuk University School of Medicine, Choongju, Korea
}

Background Temporal hollowing is inevitable after decompressive craniectomy. This complication affects self-perception and quality of life, and various techniques and materials have therefore been used to restore patients' confidence. Autologous fat grafting in postoperative scar tissue has been considered challenging because of the hostile tissue environment. However, in this study, we demonstrate that autologous fat grafting can be a simple and safe treatment of choice, even for postoperative depressed temporal scar tissue. Methods Autologous fat grafting was performed in 13 patients from 2011 to 2016. Fat was harvested according to Coleman's strategy, using a tumescent technique. Patient-reported outcomes were collected preoperatively and at 1-month and 1-year follow-ups. Photographs were taken at each visit.

Results The thighs were the donor site in all cases for the first procedure. The median final volume of harvested fat was $29.4 \mathrm{~mL}$ (interquartile range [IOR], 24.0-32.8 mL). The median final volume of fat transferred into the temporal area was $4.9 \mathrm{~mL}$ on the right side (IQR, 2.5$7.1 \mathrm{~mL})$ and $4.6 \mathrm{~mL}$ on the left side (IQR, 3.7-5.9 mL). There were no major complications. The patient-reported outcomes showed significantly improved self-perceptions at 1 month and at 1 year.

Conclusions Despite concerns about the survival of grafted fat in scar tissue, we advise autologous fat grafting for patients with temporal hollowing resulting from a previous craniectomy.

Keywords Transplantation, autologous / Adipose tissue / Transplantation / Lipectomy / Decompressive craniectomy / Quality of life
Correspondence: Hyungon Choi Department of Plastic and Reconstructive Surgery, Konkuk University Medical Center, Konkuk University School of Medicine, 120-1 Neungdong-ro, Gwangjin-gu, Seoul 05030, Korea

Tel: $+82-2-2030-7380$

Fax: +82-2-2030-5247

E-mail:md58596@hanmail.net

Received: 26 Jun 2017 - Revised: 22 Sep $2017 \bullet$ Accepted: 17 Oct 2017

pISSN: 2234-6163 • elSSN: 2234-6171 • https://doi.org/10.5999/aps.2017.01088• Arch Plast Surg 2018;45:69-73

\section{INTRODUCTION}

Decompressive craniectomy is re-emerging as a second-line therapy for patients with uncontrolled intracranial hypertension resulting from injuries or space-occupying lesions $[1,2]$. Cosmetic concerns, such as temporal hollowing, are a consequence of the resultant skull deformity.

Current theories suggest that temporal hollowing may result

Copyright (C) 2018 The Korean Society of Plastic and Reconstructive Surgeons

This is an Open Access article distributed under the terms of the Creative Commons Attribution Non-Commercial License (http://creativecommons.org/

licenses/by-nc/4.0/) which permits unrestricted non-commercial use, distribution, and reproduction in any medium, provided the original work is properly cited.

www.e-aps.org 
from atrophy of the superficial temporal fat pad due to ischemia, displacement, or denervation of the fat pad [3-5]. Kim et al. [6], in their anatomic study of the superficial temporal fat pad, proposed that temporal hollowing after coronal flap elevation may result from fat pad injury. They suggested that the superficial temporal fat pad contains a suspensory fibrous network between the superficial and deep layers of the deep temporal fascia, perforators from the deep and middle temporal arteries, and zygomaticotemporal nerve branches. They proposed that injury to these structures may lead to atrophy or subsidence of the superficial temporal fat pad, which causes temporal hollowing [6]. This can lead to an asymmetric appearance of the head, affecting patients' quality of life because of negative cosmetic perceptions by the patient or by others.

Open cranioplasty with the placement of various foreign materials is the most popular approach for augmenting the temporal fossa. Given the need for good long-term results, however, the complications inherent to foreign materials complicate their use. McNichols et al. [7] reported their experiences with augmentation temporoplasty using the classic abdominal dermal fat graft. Despite their conclusion that dermal fat grafts offered good long-term aesthetic results, the resultant scar at the donor site was a major concern for some patients, particularly if they considered their temporal deformity to be not especially severe. For patients with mild-to-moderate temporal hollowing, autogenous fat grafting can be a good alternative.

Fat grafting at previously operated sites has been considered challenging because of the hostile tissue environment. The formation of scar tissue and the scarcity of capillaries may threaten the survival of fat grafts. However, Klinger et al. [8] reported that autologous fat grafting was an efficient and safe method for treating scars of different origins, demonstrating the ability of the lipostructure technique to achieve architectural remodeling and loose connective tissue regeneration.

Accordingly, we retrospectively reviewed the medical records of patients who underwent autogenous fat grafting following decompressive craniectomy with or without cranioplasty. We found that autogenous fat grafting is a treatment option that should be considered in patients with mild-to-moderate temporal hollowing, even for postoperative temporal hollowing with ischemic soft tissue properties.

\section{METHODS}

\section{Patient selection}

We retrospectively reviewed 13 consecutive patients who underwent autogenous fat grafting at our institution for the correction of temporal hollowing resulting from decompressive crani- ectomy with or without cranioplasty between December 2011 and February 2016. The preoperative physical examination revealed the degree of temporal hollowing through a pinch between the examiner's thumb and index finger. No systematic classification of the degree of temporal hollowing exists. Although this method is subjective, thick, pliable skin with mildto-moderate adherence to the deep structure indicates that a patient may benefit from the procedure. Quantification of the preoperative temporal volume deficiency relative to the contralateral temporal area was not possible because patients were reluctant to accept the need and cost of additional radiographic evaluation. No patients underwent additional cranioplasty during successive fat grafting sessions. The patients' demographics are shown in Table 1. Preoperative photos were taken after obtaining written informed consent. In the operating room, the contour of the temporal depression was drawn with a surgical pen and visualized. All patients received 1,000 mg of cefazolin intravenously within 1 hour before the procedure.

\section{Surgical procedure}

The donor site was carefully determined preoperatively in consultation with the patient. Small incisions were made in the inguinal creases. The donor site was infiltrated with a tumescent solution, as suggested by Klein [9]. The tumescent solution was carefully infiltrated to a volume sufficient to avoid severe pain. The fat was harvested using a blunt-tip cannula $(3.0 \mathrm{~mm}$ in diameter).

According to the technique described by Coleman [10], the lipoaspirate was retained in 10-mL syringes and centrifuged at $3,000 \mathrm{rpm}$ for 2 minutes to purify the fat. After centrifugation, the fat was transferred into a $1-\mathrm{mL}$ syringe for injection, using a transducer to discard the blood and other liquid content.

Appropriate local anesthesia using a $1 \%$ lidocaine solution

\begin{tabular}{|c|c|}
\hline Characteristic & Value \\
\hline Age (yr) & $48(35-59)$ \\
\hline $\begin{array}{l}\text { Interval from previous craniectomy and/or cranioplasty } \\
\text { to fat grafting (mo) }\end{array}$ & $32.7(26.4-49.3)$ \\
\hline \multicolumn{2}{|l|}{ Subsequent cranioplasty } \\
\hline Donea) & $2(15.4)$ \\
\hline Not done & $11(84.6)$ \\
\hline \multicolumn{2}{|l|}{ Underlying conditions } \\
\hline Diabetes mellitus & $2(15.4)$ \\
\hline Hypertension & $2(15.4)$ \\
\hline Peripheral arterial occlusive disease & 0 \\
\hline Cardiopulmonary insufficiency & 0 \\
\hline
\end{tabular}


mixed with 1:100,000 epinephrine was applied to the subcutaneous layer at the planned recipient incision sites, commonly the most curved point on the patient's temporal hair line. A blunt-tip Coleman infiltration cannula was employed for injecting the fat during the withdrawal phase to avoid clustering of the fat. The fat was transplanted into the subcutaneous layer in a radial fashion, particularly in the anterior portion of the temporal hollow. Care was taken not to disrupt the temporal branch of the facial nerve and the superficial perforators running from the middle temporal artery to the superficial temporal fat pad. The fat injection was kept away from the posterior portion of the zygomatic arch to avoid injury to the perforators and the nerve branch. The temporal area was slightly overcorrected until the lateral border of the zygomaticofrontal process and the superior border of the zygomatic arch were no longer visible.

The remaining lipoaspirate was stored frozen and prepared for further sessions.

\section{Postoperative care and patient-reported outcomes}

The surface contour was molded by gentle massage only if an irregularity was noted in the intraoperative or immediate postoperative inspection. The dressing was applied with mild pressure to avoid the displacement of fat, to increase the contact area of the grafted fat parcels, and to prevent the formation of a seroma or hematoma. All patients were prescribed an oral antibiotic and non-steroidal anti-inflammatory and gastric protective medication for at least 3 days postoperatively. The patients were told not to massage the recipient site.

Patient-reported outcomes were collected using a typical 5-point Likert scale (5, very satisfied; 1 , very unsatisfied) at a preoperative visit, the 1-month follow-up visit, and the 1-year follow-up visit. For those who underwent additional fat grafting sessions, patient-reported outcomes were collected after the final procedure.

\section{Table 2. Operative procedures}

\begin{tabular}{|lc|}
\hline Characteristic & Value \\
\hline No. of procedures & \\
1 & $2(15.4)$ \\
2 & $10(76.9)$ \\
3 & $1(7.7)$ \\
Site of donation & \\
Thighs & $13(100)$ \\
Abdomen (for second procedure) & $1(7.7)$ \\
Total harvested fat (mL) & $29.4(24.0-32.8)$ \\
Total injected fat (mL) & \\
Right & $4.9(2.5-7.1)$ \\
Left & $4.6(3.7-5.9)$ \\
\hline Values are presented as number (\%) or median (interquartile range). \\
\hline
\end{tabular}

\section{Statistical analysis}

Changes in each patient's score at each follow-up point were analyzed using the Wilcoxon matched-pair test. P-values $<0.05$ were considered to indicate statistical significance.

\section{RESULTS}

The median age of the 13 patients was 48 years (range, 19-69 years). For all patients, the thighs were the only donor site for the first (or only) session. The lower abdomen was chosen as a donor site for 1 case in the second session (1 of 13, 7.7\%). In the other 12 cases, no additional harvesting was done in subsequent sessions. Ten cases (10 of $13,77.0 \%)$ required a secondary touch-up session, either at the patient's request or the surgeon's recommendation. One patient ( 1 of $13,7.7 \%)$ underwent a third and final procedure.

The median final volume of harvested fat was $29.4 \mathrm{~mL}$ (interquartile range $[\mathrm{IQR}], 24.0-32.8 \mathrm{~mL}$ ). The median final volume of fat transferred into the temporal area was $4.9 \mathrm{~mL}$ on the right side (IQR, 2.5-7.1) and $4.6 \mathrm{~mL}$ on the left (IQR, 3.7-5.9) (Table 2).

There were no major complications, such as seroma, hematoma, infection, surface irregularity, skin necrosis, or vascular compromise.

The patient-reported outcomes are shown in Table 3 and Fig. 1. At 1 month postoperatively, 92.3\% (12 of 13) of the patients were satisfied or very satisfied. At 1 year postoperatively, the proportion reporting satisfaction fell to $53.9 \%$ ( 7 of 13 ), and the proportion of neutral results increased to $30.8 \%$ (4 of 13) from $7.7 \%$ ( 1 of 13 ) at the 1 -month follow-up visit. The average score was $4.308 \pm 0.630$ at 1 month and $3.385 \pm 1.044$ at 1 year (Table $2)$. Ten patients $(76.9 \%)$ underwent a second touch-up procedure. Only 1 patient (7.7\%) required a third session.

\section{Case}

A 46-year-old female underwent decompressive craniectomy Table 3. Statistical analysis of patient-reported outcomes

\begin{tabular}{|c|c|c|c|}
\hline Likert scale & Preoperative & $\begin{array}{l}\text { 1-month } \\
\text { follow-up }\end{array}$ & $\begin{array}{c}\text { 1-year } \\
\text { follow-up }\end{array}$ \\
\hline 5 (Very satisfied) & 0 & $5(38.5)$ & $1(7.7)$ \\
\hline 4 (Satisfied) & 0 & $7(53.8)$ & $6(46.2)$ \\
\hline 3 (Neutral) & $1(7.7)$ & $1(7.7)$ & $4(30.8)$ \\
\hline 2 (Unsatisfied) & $1(7.7)$ & 0 & $1(7.7)$ \\
\hline 1 (Very unsatisfied) & 11 (84.6) & 0 & $1(7.7)$ \\
\hline P-value $e^{a)}$ & - & $0.0014^{\star \star}$ & $0.0034^{\star \star}$ \\
\hline \multicolumn{4}{|c|}{ 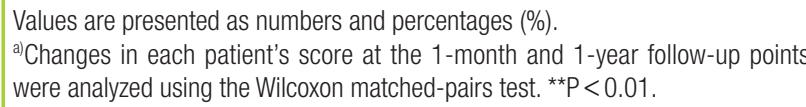 } \\
\hline
\end{tabular}


due to recurrent seizures resulting from an anaplastic oligodendroglioma in the left temporal lobe. She had postoperative left temporal hollowing for 7 years and came to our outpatient clinic. After consultation, she decided to undergo autogenous fat grafting in the depressed temporal area. A 32-mL lipoaspirate was harvested from both medial thighs and $8 \mathrm{~mL}$ was injected into the left temporal area. The remaining 24-mL lipoaspirate was stored frozen. A second touch-up session was done after the 1-month follow-up due to a remnant depression resulting from fat reabsorption. An additional $4.8 \mathrm{~mL}$ of fat was transferred at that session. She was very satisfied with the result, reporting a score of 5 on the Likert scale after the 1-month follow-up visit. The 1-year follow-up score was 4 . No donor or recipient site complications such as seroma, hematoma, infection or surface irregularity were reported (Fig. 2).

\section{Fig. 1. Patient-reported outcomes}

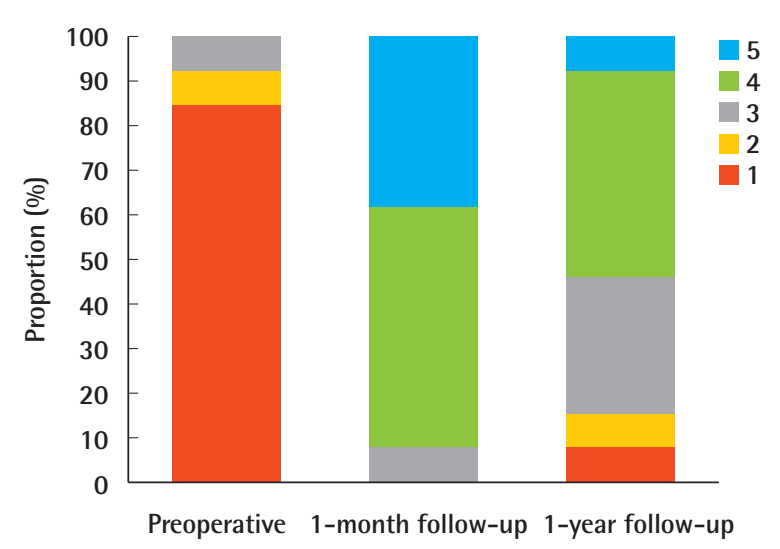

\section{DISCUSSION}

Although the exact incidence of temporal hollowing after decompressive craniectomy is unknown, temporal hollowing is nonetheless an important issue that affects patients' self-perception of their appearance and their quality of life. A variety of autogenous grafts and synthetic implants are available to correct temporal hollowing, but none of these techniques is considered the gold standard. Autogenous fat grafting possesses the advantages of simplicity and safety. It can be performed with local anesthesia in outpatient settings, unlike other grafts or implantation. Moreover, the harvested fat is intrinsically autogenous, and thus there is theoretically no risk of graft rejection or a hypersensitivity reaction. This enables significant scars at various other graft or flap donor sites to be avoided.

Fat grafting following liposuction has become popular since its introduction in 1986 by Illouz [11]. This technique has proven its simplicity and safety in many reconstructive and cosmetic applications. In 1997, Coleman [10] suggested a systematized liposuction and fat grafting procedure. This procedure, known as LipoStructure, is thought to minimize fat cell disruption and was therefore utilized to minimize resorption of the injected fat.

However, reabsorption of the grafted fat is still a limitation of this procedure. The actual survival rate of the grafted fat in the recipient environment is still unclear [12], and reabsorption may necessitate additional procedures. In our study, 10 of 13 patients $(76.9 \%)$ requested (or the surgeon suggested) an additional grafting session. Regardless of the number of times the consecutive fat graft procedures were performed, more than half of the patients (53.9\%) were satisfied with the results and 30.8\% did not perceive the need for additional sessions at the 1-year

\section{Fig. 2. A case of a 46-year-old woman}

(A) A preoperative photograph. (B) A preoperative close-up photograph. (C) A photograph at the 1-year postoperative follow-up. (D) A close-up photograph at the 1-year postoperative follow-up. Note that the shadow in the left temporal area disappeared after consecutive fat grafting procedures. The contour was acceptable and the patient was very satisfied with the result.
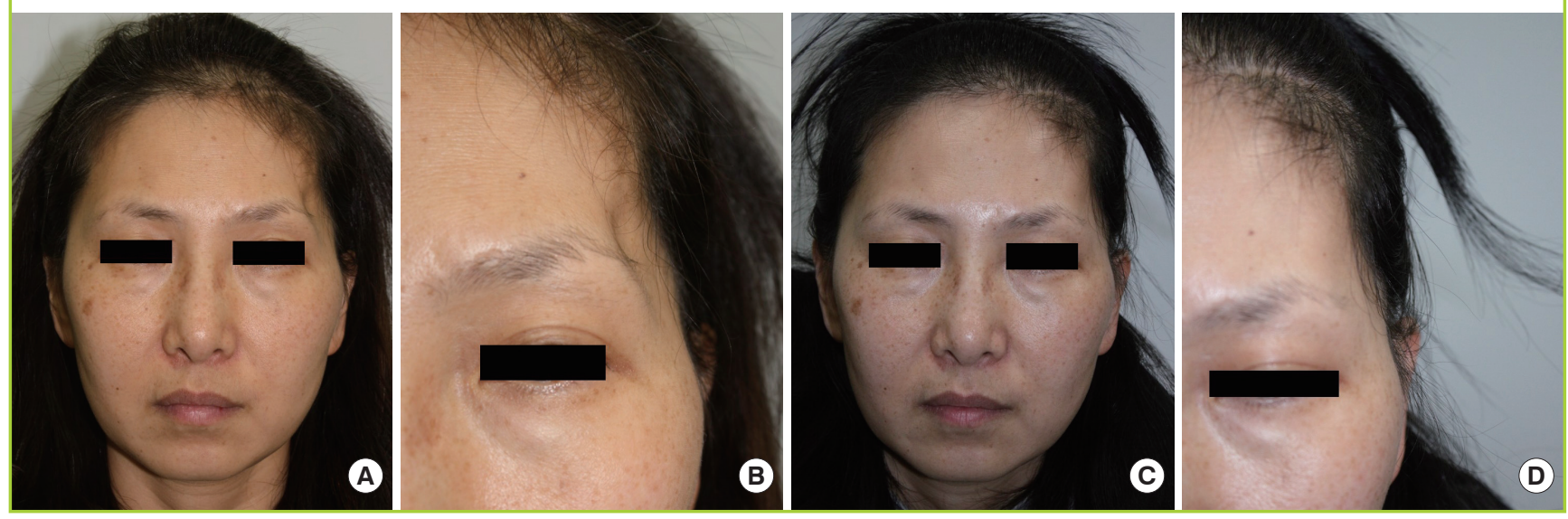


\section{follow-up.}

A healing postoperative wound or scar is generally considered a poor candidate for fat grafting because of abnormal fibrosis, hypertrophy, and the scarcity of capillaries, which are the main source of nutritional support to the grafted fat through plasmatic imbibition [13]. However, several studies have suggested that autogenous lipoaspirate containing adipose-derived stem cells has potential benefits for the regeneration of scar tissue. Rigotti et al. [14] demonstrated that treating irradiation-induced lesions with lipoaspirate transplantation was a highly effective therapeutic approach for the treatment of late side effects of oncologic radiotherapy. Klinger et al. [8] also suggested that lipofilling improved scar quality and enhanced regeneration in severe burns, other post-traumatic scars, and chronic ulcers [15]. Encouraged by Klinger's conclusion about the possible role of this procedure in post-burn scar tissue, we have performed autogenous fat grafting procedures in depressed temporal areas after craniectomy since the first patient underwent the procedure at our institution in 2011. When we retrospectively reviewed the procedures and patient-reported outcomes, we found support for Klinger's hypothesis. Despite concerns about the survival of grafted fat in scar tissue, we now consider this procedure to be a good treatment option in patients with mild-to-moderate temporal hollowing who wish to avoid major surgery under general anesthesia. Surgeons might consider this procedure as a first-line therapy because of its safety and efficacy, particularly in patients with mild-to-moderate temporal hollowing.

The current study is limited by the small number of cases and the short-term follow-up period. This may cause selection bias. The retrospective and uncontrolled nature of this study may also limit the interpretation of the results. Further prospective, randomized, controlled, quantitative, and long-term follow-up studies including more cases are needed. We did not compare our results with a control group at our institution or from other studies. Moreover, radiologic and/or histologic examinations might be useful for evaluating the rate of grafted fat survival.

\section{CONFLICT OF INTEREST}

No potential conflict of interest relevant to this article was reported.

\section{PATIENT CONSENT}

The patient provided written informed consent for the publication and the use of their images.

\section{REFERENCES}

1. Albanese J, Leone M, Alliez JR, et al. Decompressive craniectomy for severe traumatic brain injury: evaluation of the effects at one year. Crit Care Med 2003;31:2535-8.

2. Aarabi B, Hesdorffer DC, Simard JM, et al. Comparative study of decompressive craniectomy after mass lesion evacuation in severe head injury. Neurosurgery 2009;64:927-39.

3. Lacey M, Antonyshyn O, MacGregor JH. Temporal contour deformity after coronal flap elevation: an anatomical study. J Craniofac Surg 1994;5:223-7.

4. Antonyshyn OM. Soft tissue deformity after craniofacial fracture repair: analysis and treatment. J Craniomaxillofac Trauma 1999;5:19-29.

5. Persing JA, Mayer PL, Spinelli HM, et al. Prevention of "temporal hollowing" after fronto-orbital advancement for craniosynostosis. J Craniofac Surg 1994;5:271-4.

6. Kim S, Matic DB. The anatomy of temporal hollowing: the superficial temporal fat pad. J Craniofac Surg 2005; 16:7603.

7. McNichols $\mathrm{CH}$, Hatef DA, Cole P, et al. Contemporary techniques for the correction of temporal hollowing: augmentation temporoplasty with the classic dermal fat graft. J Craniofac Surg 2012;23:e234-8.

8. Klinger M, Lisa A, Klinger F, et al. Regenerative approach to scars, ulcers and related problems with fat grafting. Clin Plast Surg 2015;42:345-52.

9. Klein JA. The tumescent technique: anesthesia and modified liposuction technique. Dermatol Clin 1990;8:425-37.

10. Coleman SR. Facial recontouring with lipostructure. Clin Plast Surg 1997;24:347-67.

11. Illouz YG. The fat cell "graft": a new technique to fill depressions. Plast Reconstr Surg 1986;78:122-3.

12. Sommer B, Sattler G. Current concepts of fat graft survival: histology of aspirated adipose tissue and review of the literature. Dermatol Surg 2000;26:1159-66.

13. Smahel J. Biology of the stage of plasmatic imbibition. Br J Plast Surg 1971;24:140-3.

14. Rigotti G, Marchi A, Galie M, et al. Clinical treatment of radiotherapy tissue damage by lipoaspirate transplant: a healing process mediated by adipose-derived adult stem cells. Plast Reconstr Surg 2007;119:1409-22.

15. Klinger M, Marazzi M, Vigo D, et al. Fat injection for cases of severe burn outcomes: a new perspective of scar remodeling and reduction. Aesthetic Plast Surg 2008;32:465-9. 